\title{
Catecholaminergic polymorphic ventricular tachycardia due to de novo RyR2 mutation: recreational cycling as a trigger of lethal arrhythmias
}

Antonio Gallegos Cortez ${ }^{1}$, Néstor Alonso Ortiz², Erika Antunez Argüellez ${ }^{3}$, Teresa Villarreal Molina ${ }^{3}$, Armando Totomoch Serra ${ }^{4}$, Pedro Iturralde Torres ${ }^{5}$, Manlio F. Márquez ${ }^{5}$

\author{
${ }^{1}$ Hospital Regional de Alta Especialidad del Bajío, San Carlos la Roncha, \\ León Guanajuato, Mexico \\ ${ }^{2}$ Hospital General Naval de Alta Especialidad, Mexico \\ ${ }^{3}$ Instituto Nacional de Medicina Genómica, Mexico \\ ${ }^{4}$ Centro de Investigación y Estudios Avanzados del IPN, Mexico \\ ${ }^{5}$ Instituto Nacional de Cardiología, Mexico
}

Submitted: 26 March 2018

Accepted: 31 May 2018

Arch Med Sci 2020; 16 (2): 466-470

DOI: https://doi.org/10.5114/aoms.2019.89691

Copyright $\odot 2019$ Termedia \& Banach

Catecholaminergic polymorphic ventricular tachycardia (CPVT) is an uncommon arrhythmogenic disorder characterized by adrenergicinduced bidirectional and polymorphic VT occurring in young patients with a structurally normal heart [1-3]. Although the actual prevalence of CPVT is unknown, it is estimated at $1: 10,000$ [4]. We present the case of a 12-year-old girl suffering multiple falls while cycling finally diagnosed with CPVT and found to be heterozygous for a pathogenic RYR2 gene mutation. She did not have a family history of syncope or sudden cardiac death and was referred because of suffering multiple falls and minor trauma while riding her bicycle starting at age 8 years. Initially, her parents attributed the falls to inexperience but sought medical assistance when tonic movements of both arms occurred during one of the falls. The first clinical diagnosis was epilepsy, and, without further studies, she was treated with magnesium valproate for 6 months without clinical improvement. Due to the lack of response and the correlation of falls with exercise she was referred for evaluation to the Electrophysiology Department of the Hospital General Naval de Alta Especialidad.

On interrogation, the child (and her mother corroborated) had reduced exercise tolerance, quick fatigue, pallor, shortness of breath, and fast and irregular heartbeats associated with exercise. Physical examination was normal. The ECG showed normal P wave, PR, and QT intervals; only a fragmented QRS complex and nonspecific T wave abnormalities were observed, particularly in precordial leads, where a small notch in the descending limb of the T wave could be found (Figure 1 A). The chest X-ray and echocardiogram were normal. Holter monitoring (Figure 1 B) showed frequent ventricular premature beats (VPB) with multiple morphologies (14\%, $n=1603$ ), and presence of couplets and triplets (pleomorphic), reproducible with heart rates above $110 \mathrm{bpm}$. Also, a self-limited supraventricular tachycardia at $261 \mathrm{bpm}$ was documented.

In an exercise stress test (Bruce protocol; Figure 2) a monomorphic VPB occurred when the heart rate reached $100 \mathrm{bpm}$. Ventricular premature beats in bigeminy and pleomorphic couplets were observed shortly after, then bidirectional VT at 200 bpm occurred, and a brief episode

\author{
Corresponding author: \\ Antonio Gallegos Cortez \\ Hospital Regional de Alta \\ Especialidad del Bajío \\ San Carlos la Roncha \\ 37660, León Guanajuato \\ Mexico \\ E-mail: \\ antonio_gallegoscortez@ \\ yahoo.es
}


A

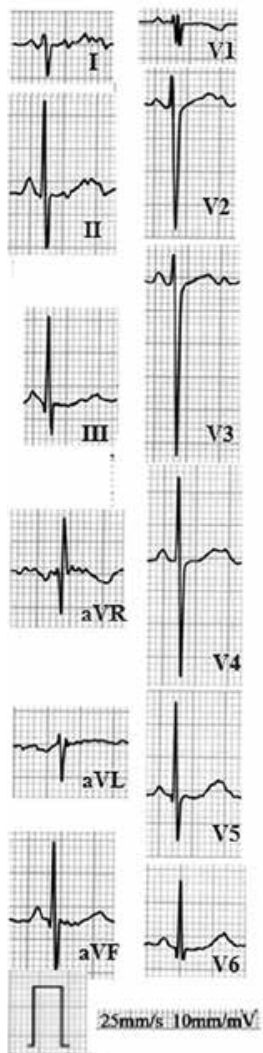

B

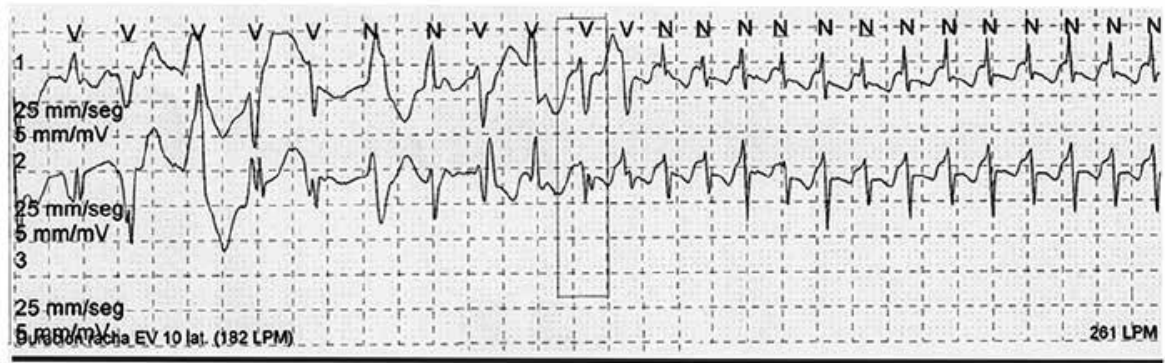

$-N-N-N-N-N-N-N-N-N-N-N-N-N-N-N-N-N-N-N-N-N-N-N-N-N-N-N-N$ $25 \mathrm{~mm} / \mathrm{seg}^{-2}$

$5 \mathrm{~mm} / \mathrm{mV} \ldots$ $25 \mathrm{~mm} / \mathrm{seg}$
$5 \mathrm{~mm} / \mathrm{mV}$

$25 \mathrm{~mm} / \mathrm{seg}$

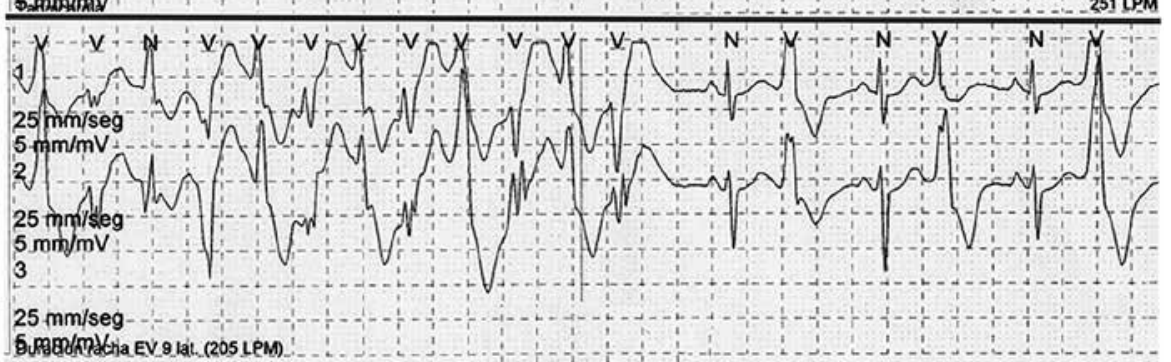

Figure 1. A - 12-lead ECG $(25 \mathrm{~mm} / \mathrm{s}, 10 \mathrm{~mm} / \mathrm{mV})$. B - Holter monitoring. The first strip shows the beginning of the non-sustained narrow QRS tachycardia that continues in the second strip. On the third strip, bidirectional ventricular tachycardia is followed by bigeminy

of polymorphic VT (7 beats) was recorded just before the exercise was stopped. These findings were associated with pallor, diaphoresis, and dyspnea. Paroxysmal supraventricular tachycardia was documented in the recovery phase. An electrophysiology study was performed. No ventricular or supra-ventricular arrhythmias were induced despite programmed stimulation from the right atrium, coronary sinus, and right ventricular apex (with single and double ventricular extra-stimuli at two different driven cycle lengths: standard Josephson protocol). Single nodal physiology was demonstrated. Absence of an accessory pathway was confirmed. No pharmacological challenge (isoproterenol, dobutamine) was performed.

With the primary diagnosis of CPVT, the patient was referred for genetic testing. Genomic DNA was extracted from whole blood. In the first instance, previous assent from the patient and permission of their parents was obtained, and an informed consent form was signed and collected after an explanation of the risks, limitations and achievements of the genetic testing, including the consideration in the future for both parents to carry out confirmatory studies, if they would have the will to participate.
Genetic testing was performed using a targeted sequence Haloplex custom design (Agilent Technologies, Santa Clara, CA, USA) including 83 cardiogenes, in a MiSeq device (Illumina, San Diego, CA, USA). Bioinformatic analyses (alignment and variant calling) were performed using BaseSpace (Illumina), and variants were annotated using ANNOVAR (http://wannovar.wglab.org/). Variants were filtered for quality, impact, and functional consequences according to SIFT (http://sift.jcvi.org/), Polyphen-2 (http://genetics.bwh.harvard.edu/pph2/), Mutation Taster (http://www.mutationtaster.org/), FATHMM (http://fathmm.biocompute.org.uk/) and Provean (http://provean.jcvi.org/ index. php). The analysis identified a variant that affects function (c.11836G>A, rs794728777, or p.Gly3946Ser mutation) in the $R Y R 2$ gene in a heterozygous state. The mutation was verified in the patient and tested in both parents by Sanger sequencing in an $A B I$ PRISM 3100 genetic analyzer (ABI 3100, Applied Biosystems, Foster City, CA). Only the patient carried the p.Gly3946Ser mutation in a heterozygous form, suggesting a de novo origin (Figure 3).

The patient was treated with propranolol up to $1 \mathrm{mg} / \mathrm{kg} /$ day. After only 6 days of pharmacological treatment, a significant reduction in arrhythmic 


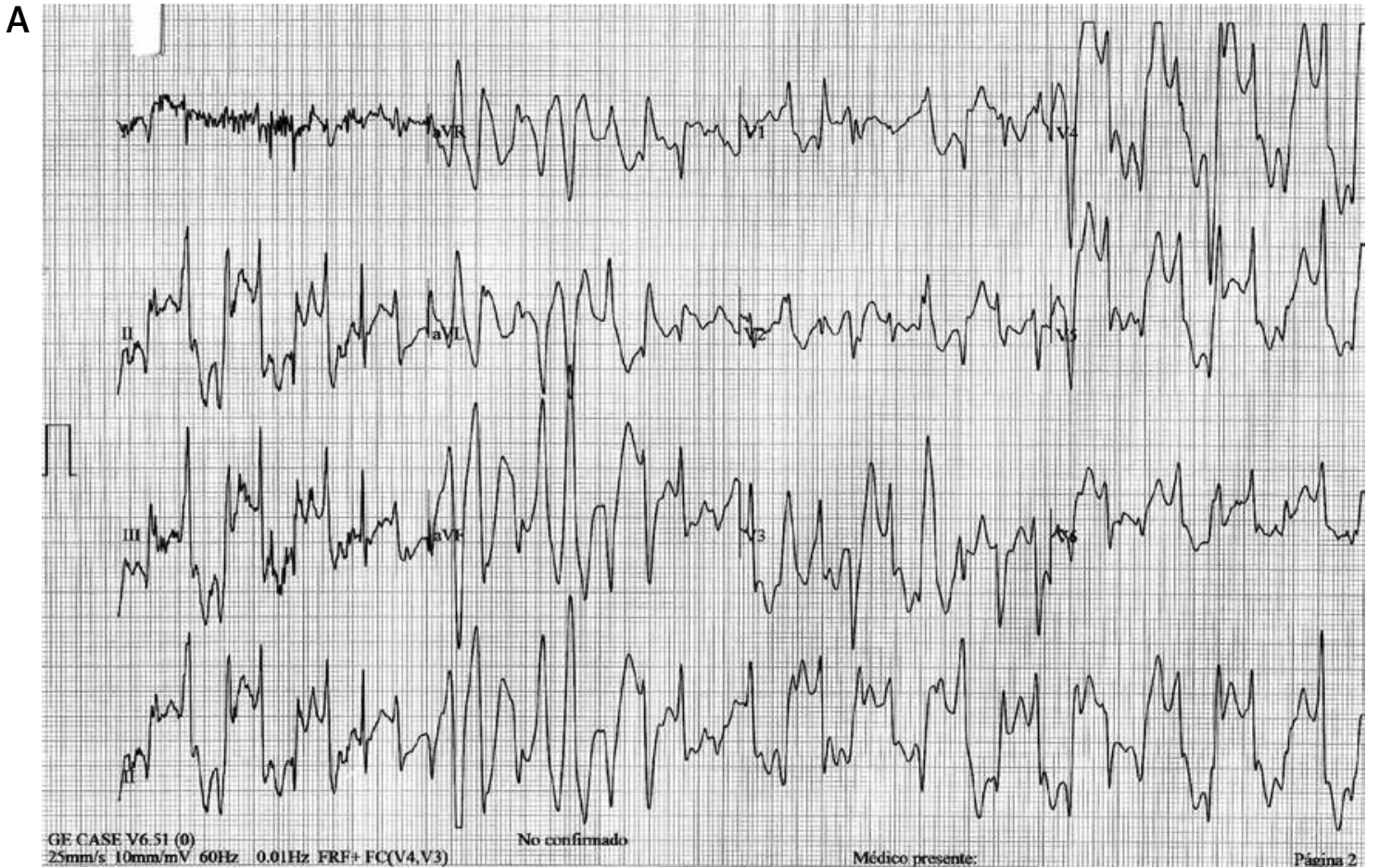

B

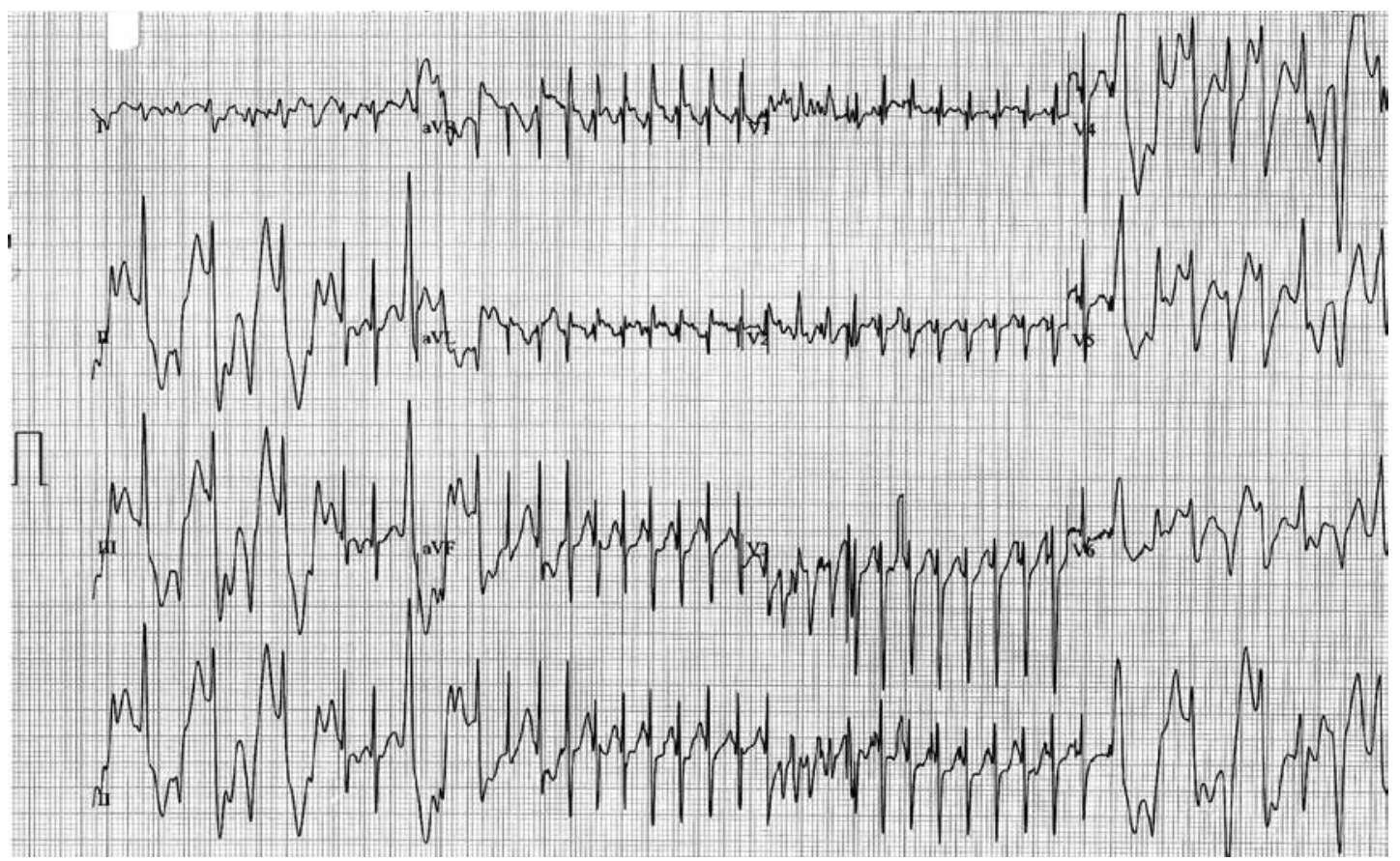

Figure 2. Exercise stress testing. The first strip shows the polymorphic ventricular tachycardia preceded by bidirectional ventricular tachycardia (at $52 \mathrm{~s}$ of the first stage). The recovery phase is presented on the second strip where a non-sustained narrow QRS tachycardia is observed

load was observed, with only a few isolated monomorphic VPB and couplets in Holter monitoring (276 VPB/24 h (0.3\%)). Longer exercise time was achieved on a second exercise stress test (with the same Bruce protocol); VPB and bigeminy were only observed after the heart rate reached $120 \mathrm{bpm}$. The test was terminated when the first couplet was identified, at the first minute of phase 4 (for energy consumption of $12 \mathrm{MET}$ ). The patient experienced no symptoms during exercise. At 57-month follow-up, the girl remains asymptomatic and with no recurrence of syncope or palpitations.

To date, mutations in four different genes have been reported to cause CPVT [5], and also in the 
A

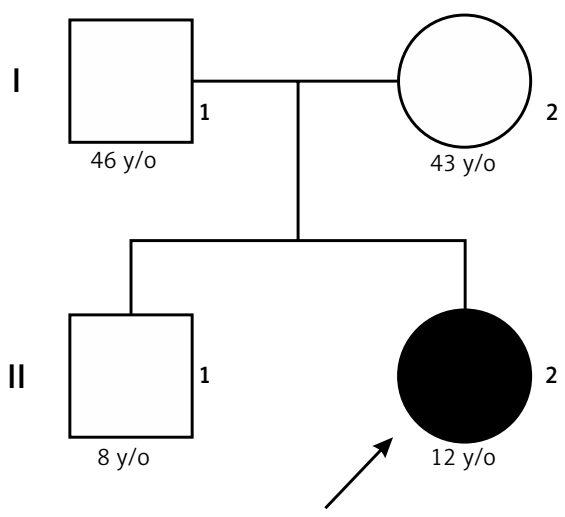

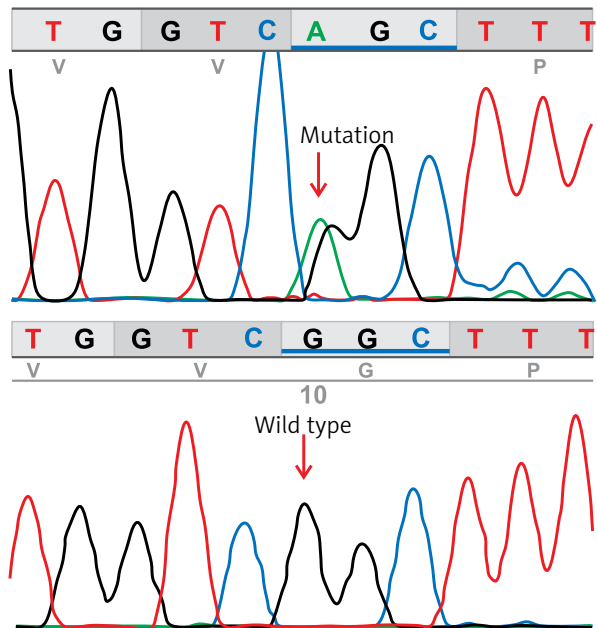

Figure 3. A - Family pedigree. Both parents and sibling are unaffected and do not carry the c.11836G $<\mathrm{A}$ mutation. B - Electropherogram of the proband, showing the missense c.11836G<A mutation (p.Gly3946Ser) in exon 88 of the $R Y R 2$ gene

RYR2 gene (encoding the cardiac ryanodine receptor channel) $[6,7]$ mutations are found in 60-65\% of CVPT patients and are transmitted in the autosomal dominant pattern. CALM1 (encoding calmodulin) [8], TRDN (encoding triadin) [9], or CASQ2 (encoding cardiac calsequestrin) [10] may also be the cause of CPVT.

The key feature underlying pathogenesis of CPVT is the aberrant release of $\mathrm{Ca}^{2+}$ into the sarcoplasmic reticulum during diastole (termed a transient inward current) leading to diastolic $\mathrm{Ca}^{2+}$ leak, which provides a substrate for delayed afterdepolarizations (DADs), specifically in the setting of $\beta$-adrenergic stimulation during stress or exercise. It has been proposed that CPVT-causing RYR2 mutations result in a gain-of-function of the ryanodine receptor leading to a diastolic $\mathrm{Ca}^{2+}$ leak [2, 11, 12].

Massive parallel sequencing helped identify a de novo missense RYR2 mutation C.11836G $>\mathrm{A}$ (p.Gly3946Ser) in the patient (Figure 3 B), located in one of the mutational hotspots of this gene. Annotation showed that the variant is predicted as damaging or deleterious by more than 5 in silico functional prediction programs. It is considered as pathogenic in the ClinVar database (www.ncbi.nlm. nih.gov/clinvar/variation/201315/), and has been previously reported in other patients with CPVT [7]. Although the mutation has not been functionally characterized in vitro, it is considered as pathogenic according to the criteria of Campuzano [13].

The case here reported exemplifies the clinical picture of CPVT in a child who suffered exerciseinduced syncope misdiagnosed as seizures. She had a history of multiple falls while cycling. Initially, her parents were not concerned, believing the child was unskilled, but became concerned when they observed tonic extremity movements during the falls. As frequently occurs in hereditary sudden cardiac death syndromes, the child was misdiagnosed with epilepsy and treated with anticonvulsant drugs, showing no clinical response [14]. Between $20 \%$ and $30 \%$ of patients undergoing long-term follow-up in hospital epilepsy clinics do not have epilepsy [15]. In this case a more detailed initial clinical evaluation would have revealed that pseudoseizures were indeed related to exercise and raise suspicion of a cardiac syncope. It has been reported that a noninvasive cardiovascular evaluation including head-up tilt test, carotid sinus massage electroencephalography, and blood pressure monitoring could identify an alternative diagnosis in up to $41 \%$ of patients with apparent epilepsy, mainly vasovagal syncope [16]. However, confirming that convulsive syncope is the result of a cardiac arrhythmia is not an easy task. Previous observational studies and case reports have shown that prolonged QT and Brugada syndrome should be suspected in children who experience seizures for the first time.

The present case indicates that this is also true for CPVT [17]. The neurological investigation is insufficient if it does not include cardiological evaluation $[18,19]$ as previously stated. Failure to perform a cardiological evaluation may lead to severe consequences. Sudden death occurs in up to $33 \%$ of CPVT patients, and almost $80 \%$ experienced cardiac events before age 40 years, despite treatment [1]. Sports competition and extreme physical exercise are contraindicated in these patients. Beta-blockers are the first-line treatment and flecainide can be added if necessary. In selected cases, mainly those unresponsive to drugs, implant- 
ed cardiac defibrillator or left sympathectomy can be considered [20]. Propranolol treatment at $1 \mathrm{mg} / \mathrm{kg} /$ day led to an essential reduction of VPB (from $14 \%$ to $0.3 \%$ ) in our patient. With pharmacological treatment only, she has remained asymptomatic after a 57-month follow-up.

Finally, concerning the genetic testing, the direct contact (instead of family contact) with both parents was the best method to ensure the later participation in the cascade screening after the identification of the mutation in the patient [21]. Because the mutation was found only in the index case, it most likely occurred de novo. However, gonadal mosaicism in one of the parents, while highly unlikely, cannot be wholly ruled out. Furthermore, $30 \%$ of patients with CPVT had sudden cardiac arrest as first presentation [22] Because of this, genetic testing is useful to identify those patients with an apparent negative phenotype in cascade screening, mainly for carriers of mutations in RyR2 and CASQ2 [20]. A negative test does not exclude the clinical management in a suspected case of CPVT, even in cases of phenocopy or an unknown gene. Incidental findings can occur with the use of massive parallel sequencing, and in all cases, the results of the test must be provided in a clinical context, by healthcare professionals with expertise in the area.

In conclusion the case herein reported exemplifies (1) the diagnostic challenge of hereditary sudden death syndromes and utility of genetic testing; (2) light exercise (recreational cycling) as a trigger for a malignant arrhythmia; (3) an excellent response to $\beta$-blocker treatment alone in CPVT.

\section{Conflict of interest}

The authors declare no conflict of interest.

\section{References}

1. Napolitano C, Priori SG. Diagnosis and treatment of catecholaminergic polymorphic ventricular tachycardia. Heart Rhythm 2007; 4: 675-8.

2. Rueda A, de Alba-Aguayo DR, Valdivia HH. Ryanodine receptor, calcium leak and arrhythmias. Arch Cardiol Mex 2014; 84: 191-201.

3. González-Melchor L, Villarreal-Molina T, Iturralde-Torres P, Medeiros-Domingo A. Muerte súbita cardiaca en el corazón estructuralmente normal: una actualización. Arch Cardiol Mex 2014; 84: 293-304.

4. Napolitano C, Priori SG, Bloise R. Catecholaminergic polymorphic ventricular tachycardia. 2004 Oct 14 [Updated 2014 Mar 6]. In: Pagon RA, Adam MP, Ardinger HH, et al. (eds.). GeneReviews ${ }^{\oplus}$ [Internet]. University of Washington, Seattle 1993-2015. Available from: http://www.ncbi. nlm.nih.gov/books/NBK1289.

5. Ackerman MJ, Priori SG, Willems S, et al. HRS/EHRA expert consensus statement on the state of genetic testing for the channelopathies and cardiomyopathies: this document was developed as a partnership between the Heart Rhythm Society (HRS) and the European
Heart Rhythm Association (EHRA). Europace 2011; 13: 1077-109.

6. Laitinen PJ, Brown KM, Piippo K, et al. Mutations of the cardiac ryanodine receptor (RyR2) gene in familial polymorphic ventricular tachycardia. Circulation 2001; 103: 485-90.

7. Priori SG, Napolitano C, Tiso N, et al. Mutations in the cardiac ryanodine receptor gene (hRyR2) underlie catecholaminergic polymorphic ventricular tachycardia. Circulation 2001; 103: 196-200.

8. Nyegaard M, Overgaard MT, Søndergaard MT, et al. Mutations in calmodulin cause ventricular tachycardia and sudden cardiac death. Am J Hum Genet 2012; 91: 703-12.

9. Roux-Buisson N, Cacheux M, Fourest-Lieuvin A, et al. Absence of triadin, a protein of the calcium release complex, is responsible for cardiac arrhythmia with sudden death in human. Hum Mol Genet 2012; 21: 2759-67.

10. Lahat $H$, Pras E, Olender T, et al. A missense mutation in a highly conserved region of CASQ2 is associated with autosomal recessive catecholamine-induced polymorphic ventricular tachycardia in Bedouin families from Israel. Am J Hum Genet 2001; 69: 1378-84.

11. Behere SP, Weindling SN. Catecholaminergic polymorphic ventricular tachycardia: an exciting new era. Ann Pediatr Cardiol 2016; 9: 137-46.

12. Priori SG, Napolitano C, Memmi M, et al. Clinical and molecular characterization of patients with catecholaminergic polymorphic ventricular tachycardia. Circulation 2002; 106: 69-74.

13. Campuzano O, Allegue C, Fernandez A, Iglesias A, Brugada R. Determining the pathogenicity of genetic variants associated with cardiac channelopathies. Sci Rep 2015; 5: 7953.

14. Hazle MA, Shellhaas RA, Bradley DJ, Dick M $2^{\text {nd }}$, Lapage MJ. Arrhythmogenic channelopathy syndromes presenting as refractory epilepsy. Pediatr Neurol 2013; 49: 134-7.

15. Zaidi A, Clough P, Cooper P, Scheepers B, Fitzpatrick AP. Misdiagnosis of epilepsy: many seizure-like attacks have a cardiovascular cause. J Am Coll Cardiol 2000; 36: 181-4.

16. Choong H, Hanna I, Beran R. Importance of cardiological evaluation for first seizures. World J Clin Cases 2015; 3: 381-4.

17. Liu X, Li XL, Hu JZ, et al. Recurrent syncope related to catecholaminergic polymorphic ventricular tachycardia due to de novo RyR2-R2401H mutation. Zhonghua Xin Xue Guang Bing Za Zhi 2017; 45: 39-43.

18. Kostera-Pruszczyk A, Miszczak-Knecht M, Ciurzyński M, Pruszczyk P. Propafenone is not effective for severe ventricular arrhythmias in Andersen-Tawil syndrome. Arch Med Sci 2018; 14: 248-50.

19. Morosin M, Dametto E, Del Bianco F, Brieda M, Nicolosi GL. An unusual etiology of torsade de pointes-induced syncope. Arch Med Sci 2017; 13: 686-8.

20. Priori SG, Wilde AA, Horie M, et al. HRS/EHRA/APHRS expert consensus statement on the diagnosis and management of patients with inherited primary arrhythmia syndromes: document endorsed by HRS, EHRA, and APHRS in May 2013 and by ACCF, AHA, PACES, and AEPC. Heart Rhythm 2013; 10: 1932-63.

21. Newson AJ, Humphries SE. Cascade testing in familial hypercholesterolaemia: how should family members be contacted? Eur J Hum Genet 2005; 13: 401-8.

22. Priori SG, Napolitano C, Memmi M, et al. Clinical and molecular characterization of patients with catecholaminergic polymorphic ventricular tachycardia. Circulation 2002; 106: 69-74. 